\title{
Tissue expression of S100 proteins in gallbladder mucosa of the patients with calculous cholecystitis
}

\author{
Miroslaw Szmyt $^{1}$, Aldona Kasprzak ${ }^{1}$, Wojciech Malkowski ${ }^{2}$, Joanna Surdyk-Zasada ${ }^{1}$, \\ Wieslawa Przybyszewska ${ }^{1}$, Elzbieta Siodla ${ }^{1}$, Agnieszka Seraszek-Jaros ${ }^{3}$, \\ Joanna Jagielska ${ }^{3}$
}

\author{
${ }^{1}$ Chair and Departament of Histology and Embryology, Poznan University of Medical Sciences \\ ${ }^{2}$ Chair of General Surgery T. Chalubinski Municipal Hospital in Ostrow Wielkopolski \\ ${ }^{3}$ Department of Bioinformatics and Computational Biology in the Chair of Clinical \\ Pathomorphology, Poznan University of Medical Sciences
}

\begin{abstract}
Proteins of S100 group, produced by phagocytes represent endogenous activators of innate immune responses. Role of these proteins in the etiopathogenesis of cholelithiasis remains unknown. The studies aimed at the morphometric evaluation of S100A8 and S100A9 protein expression in gallbladder mucosa in patients with acute and chronic calculous cholecystitis $(\mathrm{n}=71)$. The presence of proteins was detected by immunohistochemistry while quantitative analysis employed the spatial visualization technique. We found the immunopositive expression of the two studied S100 proteins in neutrophils and monocytes/macrophages of the gallbladder's wall and a higher expression in acute cholecystitis. Quantitative study revealed higher immunoexpression of S100A9 over S100A8 in both studied groups of patients. Moreover, a reciprocal linear relationship between the expression of the studied proteins and a positive correlation between expression of either S100A8 or S100A9 and inflammatory activity (grading) in the gallbladder wall were found. The expression of S100A8 protein in the chronic cholecystitis group and in older patients correlated with leukocytosis, which suggests the role of S100A8 particularly at the chronic stage of cholecystitis. The obtained results indicated close relationship between S100A8 and S100A9 proteins in their proinflammatory functions. The increased expression of only one of them can be recognized as a useful index of local inflammatory activity in calculous cholecystitis. (Folia Histochemica et Cytobiologica 2013, Vol. 51, No. 2, 141-148)
\end{abstract}

Key words: calculous cholelithiasis, gallbladder wall, S100A8, S100A9, calgranulin, age, semiquantitative immunohistochemistry

\section{Introduction}

S100 proteins belong to the family of 25 molecules with mostly low molecular weight (9-14 kDa), high homology and a tissue-specific expression [1-3]. The group of S100 proteins contains a subgroup of prote-

Correspondence address: A. Kasprzak, Department of Histology and Embryology

Poznan University of Medical Sciences;

Swiecickiego St. 6, 60-781 Poznan, Poland;

tel.: +48 6185464 41; fax: +48 6185464 40;

email: akasprza@ump.edu.pl ins (S100A8, S100A9 and S100A12) strictly linked to the control of immune system, the overexpression of which was observed in acute and chronic inflammatory diseases and tumours [4-6]. Similarly to most of the S100 family proteins, S100A8 and S100A9 proteins may manifest as homodimers but they preferentially form S100A8/A9 heterodimers or tetramers $[1,3,5,6]$. S100A8 and S100A9 proteins, produced and secreted mainly by stimulated neutrophils, activated monocytes and macrophages, show strong chemotactic activity [4]. A positive correlation was documented between extracellular concentration of the proteins, number of neutrophils and loss of the cells viability [7]. On the 
other hand, S100A8 and S100A9 proteins, present in activated endothelial cells, fibroblasts and keratinocytes, play mainly intracellular roles. S100A8 protein is termed also as calgranulin A (or myeloid-related protein 8, MRP8), S100A9 protein has an alternate name of calgranulin B (or MRP14) and S100A12 protein of calgranulin C (or EN-RAGE, extracellular newly identified RAGE-binding protein) [7, 8]. S100A8/ /A9 heterodimer accounts for $40-50 \%$ of the soluble cytosol fraction of neutrophils [1,9]. It participates in the innate resistance and transendothelial migration of leukocytes to sites of inflammation and is regarded as an indicator of chronic organ inflammation $[4,5,8]$.

S100A8/S100A9 heterodimer and other proteins of the S100 family (S100B, S100A12, S100P) bind at the plasma membrane of target cell to multiple receptors and/or coreceptors, including heparan sulphate and carboxylated glycans [10], CD36 and receptor of advanced glycation end products (RAGE) [2]. S100A8 and S100A9 proteins represent probable ligands also of the Toll-like receptor 4 (TLR-4) [11].

In inflammatory infiltrates of neutrophils and/ /or activated monocytes/macrophages elevated levels of S100A8 and S100A9 were detected, also in the systemic circulation $[6,12]$. The S100A8/A9 complex present in faeces („faecal” calprotectin) was suggested to represent a marker of intestinal inflammatory conditions [5, 13]. The presence of S100A8/S100A9 proteins was demonstrated by immunocytochemical (IHC) method in phagocytes of inflammatory infiltrates of small intestine [14]. In chronic intestinal diseases (active Crohn's disease, ulcerative colitis, Irritable Bowel Syndrome) increased secretion was reported of mainly S10012 protein and a less accentuated one of S100A8/A9 proteins both in adults and children [5, 15]. Presence of S100A8/A9 and S100A12 proteins was demonstrated in gastric mucosa of children with Helicobacter pylori infection [16]. On the other hand, augmented expression of S100B protein was demonstrated in patients with untreated coeliac disease [17]. It was found that $\mathrm{S} 100 \mathrm{~B}$ participated in the induction of nitrogen oxide synthase and nitrite production [17]. Exposure to gliadin markedly intensified production of S100B protein [17]. Increased tissue expression of S100A9 and S100A8 was demonstrated also in $70 \%$ of colorectal carcinoma patients [18], while tissue expression of S100A4 in gallbladder carcinoma was found to represent a negative prognostic factor in this type of tumour [19].

Although S100A8 and S100A9 proteins play mainly a proinflammatory role, their other functions (including anti-inflammatory activity) may result from post-translational modifications of proteins, activity of proteases released from activated phagocytes, binding of zinc ions, and other not yet fully recognized factors [6]. The elucidation of various activities of S100A8 and S100A9 proteins, produced by various human cell populations, requires further studies, particularly in the context of their diagnostic or therapeutic applications.

Role of the S100 proteins produced by phagocytes in the pathogenesis of gallbladder cholelithiasis and cholecystitis has been poorly recognized and no respective data are available in the accessible publications. It also remains unknown whether, similarly to other diseases of the alimentary tract, these proteins may serve as markers of gallbladder inflammation which accompanies cholelithiasis. Therefore, this study aimed at the evaluation of the cellular expression of the S100 protein group in the gallbladder mucosa of the patients with calculous cholecystitis and an attempt was made to correlate the expression with selected clinical data of the patients.

\section{Material and methods}

Patients. All the patients were subjected to cholecystectomy in the T. Chalubinski Municipal Hospital in Ostrow Wielkopolski. The group with acute calculous cholecystitis (ACC) ( $n=16 ; 7$ women and 9 men) and chronic calculous cholecystitis (CCC) $(\mathrm{n}=55$; 44 women and 11 men $)$ included patients who were diagnosed and subjected to surgery in years 2003-2007. The CCC group was divided into two subgroups: patients up to 25 years of age $(n=35$, subgroup $A)$ and patients older than 50 years $(n=20$, subgroup $B)$. Exclusion criteria involved patients who had previously past experienced other abdominal operations than cholecystectomy, patients with systemic diseases of connective tissue, patients with alcohol abuse, patients who chronically used anti-inflammatory drugs or other drugs which might modify the process of wound healing or inflammatory process itself.

Duration of the cholelithiasis symptoms in the analysed groups of patients most frequently ranged between 6 months and one year. The available epidemiological data included age, gender and body mass index (BMI) upon admission to the hospital. The other available variables included results of laboratory/ /biochemical tests. The number of gallstones disclosed following cholecystectomy was estimated using a semiquantitative scale: 1 - a single gallstone of any size; $2-2$ to 20 gallstones; $3>20$ to 100 gallstones; $4>100$ gallstones. In both groups of patients mostly mixed, cholesterol/pigment/calcium gallstones were detected. However, their detailed chemical composition was not tested. Written informed consent was obtained from each patient before operation, and approval for the study was granted by the institution's Ethical Committee.

Tissue material and microscopy image analysis. The archival material was fixed in a buffered $10 \%$ formalin and embedded in paraffin using the routine procedure. 
The studies were conducted on serial, $5 \mu \mathrm{m}$-thick sections placed on the SuperFrost/Plus microscope slides. Slides were stained with hematoxylin and eosin (HE), and patterns of HE-stained gallbladder histological preparations were examined independently by two histopathologists (WB, AK). Thickness (width) of the total wall of gallbladder was measured in mm. Each tissue specimen was also evaluated based on a simple numerical scoring system for the grade of lamina propria inflammation (G1) (0-3), and the grade of muscularis externa/adventitia inflammation (G2) (0-3), in which 3 points denoted an intense and, most frequently, diffuse inflammatory infiltrate, 2 points referred to moderately intense but also diffuse inflammatory infiltrate, 1 point indicated single, dispersed cells or focally arranged cells of inflammatory infiltrate, 0 points indicated tissue sections in which no cells of inflammatory infiltrate could be detected.

Immunocytochemical studies. Detection of cellular localization of S100A 8 and S100A9 proteins in gallbladder mucosa were performed by the classic $\mathrm{ABC}$ (streptavidin-biotinylated peroxidase complex) method according to Hsu et al. [20], the individual stages of which were described in detail in our earlier report [21]. Following deparaffination and rehydration slides were additionally boiled in $10 \mathrm{mM}$ citrate buffer in microwave oven at $700 \mathrm{~W}$ for $18 \mathrm{~min}$. to increase antigen retrieval, washed in PBS and, then, subjected to standard IHC procedure. Rabbit anti-human S100A8 and anti-human S100A9 polyclonal antibodies were employed (SigmaAldrich, Poznan, Poland), both in dilution 1:5000. The sections were incubated with these primary polyclonal antibodies overnight at $4^{\circ} \mathrm{C}$, and afterwards secondary biotinylated link anti-mouse and anti-rabbit $\mathrm{IgG}$ with the $\mathrm{ABC}$ kit (Dako REAL ${ }^{\mathrm{TM}}$ Envision $^{\mathrm{TM}}$, Dako, Glostrup, Denmark) were consecutively applied. The colour reaction was evoked with the HRP substrate, $0.05 \%$ 3,3-diaminobenzidine tetrahydrochloride (DAB; DAKO) in $0.05 \mathrm{M}$ Tris- $\mathrm{HCl}$ buffer, $\mathrm{pH}$ 7.6, supplemented with $0.001 \% \mathrm{H}_{2} \mathrm{O}_{2}$. In negative controls specific antibodies were omitted and supplemented by a normal serum of a respective species in $0.05 \mathrm{M}$ Tris- $\mathrm{HCl}, \mathrm{pH} \sim 7.6$, supplemented with $0.1 \%$ bovine serum albumin (BSA) and $15 \mathrm{mM}$ sodium azide. Identical IHC technique was applied to the sections of archival reactive lymph nodes $(n=6)$ as a positive control. However, studies in this control group aimed only at verification of quality of the applied antibodies and were not subjected to statistical analysis.

Histological slides were examined with Olympus BH-2 microscope coupled to a digital camera. Colour microscope images were recorded and archived using $40 \times$ objective (at least 10 fields in every slide with an immunopositive reaction in the gallbladder mucosa), using LUCIA Image 5.0 computer software.

Microscopy image analysis and statistical analysis. The quantitative evaluation of the immunocytochemical expression of the proteins in gallbladder mucosa was performed using image processing method based on spatial visualization of markers in microscope images, elaborated and programmed in the A4D computer software $\mathrm{C}++$ language by Kaczmarek and Strzelczyk [22] and described in details in our previous paper [23]. The A4D programme allowed to sequentially calculate: (1) mean surface area of the entire mucosa (epithelium + lamina propria) in the recorded image (in pixels and $\mu \mathrm{m}^{2}$ ) for every patient and for the entire group of patients, (2) mean surface area of the IHC reaction (detecting presence of S100A8 and S100A9) per the entire gallbladder mucosa (in pixels and $\mu \mathrm{m}^{2}$ ) for every patient, (3) mean surface area of immunocytochemical reaction for a S100A8 and S100A9 per entire mucosa in every patient, calculated and expressed also as percentages $(\%)$ of the whole mucosal surface.

Results obtained in the two softwares for image analysis (LUCIA Image 5.0 and A4D) were exported to the Microsoft Excel programme, and further were statistically analysed with the Statistica PL v. 8 programme (Statsoft, Inc., Tulsa, OK, USA). In this study results related to expression of proteins were presented as area fraction (percentage) of the IHC reactions manifested by the mucosa (epithelium + lamina propria). The Shapiro-Wilk normality test was performed to determine whether the obtained results were normally distributed. A significance of differences between acute calculous cholecystitis (ACC) and chronic calculous cholecystitis (CCC) groups and the younger (subgroup A) and older patients (subgroup B) from CCC group was evaluated using the $t$ test. Subsequently, parameters of a descriptive statistics were calculated (arithmetical mean, standard deviation, median value, minimum and maximum values). Results of microscopic image analysis were compared between the ACC and CCC independent group of the patients using the Mann-Whitney U test and the Wilcoxon test for non-parametric dependent data. Correlations between data rows were determined employing linear Pearson or Spearman's rank correlation index. Differences and relationships were accepted to be statistically significant at the level of $\mathrm{p}<0.05$.

\section{Results}

\section{Cellular localization of S100A8 and S100A9 proteins}

S100A8 and S100A9 proteins were detected by immunohistochemistry in gallbladder mucosa of all patients. Both proteins were mainly localized in the cytoplasm of the inflammatory infiltrates in lamina propria (mainly neutrophils and monocytes/macrophages) (Figure 1A). Positive reaction was detected also in the epithelial cells of the gallbladder (Figure 1B) and in vascular endothelium (Figure 1C). Immunopositive cells were detected also in the lumen of blood vessels. In patients of both ACC and CCC 

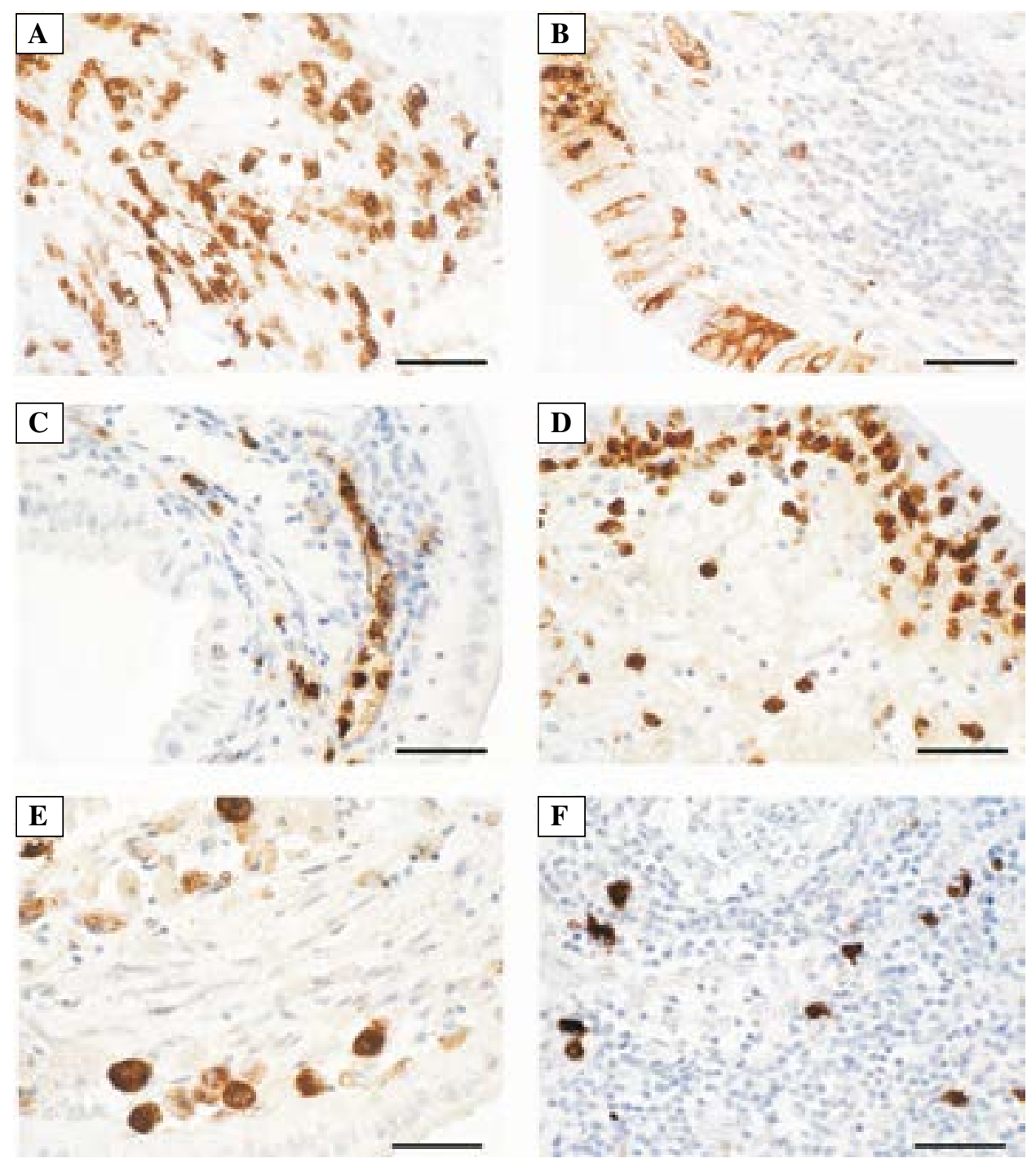

Figure 1. S100A8 and S100A9 expression in the gallbladder mucosa of patients with cholelithiasis (A-E). Numerous S100A8-positive neutrophils and monocytes/macrophages in gallbladder lamina propria (A); S100A9 protein in gallbladder epithelial cells (B) and S100A8 protein in vascular endothelium (C); S100A9-positive cells infiltrating gallbladder epithelium (D) and localized in foamy cells within lamina propria (E). Only single S100A8-positive cells in the reactive lymph node (positive control) were noted (F). ABC technique. Hematoxylin counterstained; Bar $=40 \mu \mathrm{m}$

groups expression of both proteins was also noted in cells infiltrating gallbladder epithelium (Figure 1D). In the CCC group S100A9 protein was localized also in a few foamy cells within lamina propria (Figure 1E).

In the reactive lymph node (positive control) only single S100A8- and S100A9-positive cells could be observed (Figure 1F).

\section{Semiquantitative analysis of S100A8 and S100A9 proteins expression}

In the mucosa of the ACC patients mean expressions (percentage of the immunopositive reactions) of S100A8 and S100A9 proteins were significantly higher than in CCC patients (Figures 2 and 3). In the case of S100A8 higher expression of the protein was demonstrated in older as compared with younger patients of the CCC subgroup (Figure 2).

Moreover, in respect to the two groups of patients with acute and chronic type of cholecystitis (ACC and CCC, respectively) the expression of S100A9 protein proved to be higher than that of S100A8 protein (Figures 4 and 5).

\section{Correlation between reciprocal expression of S100A8 and S100A9 proteins}

In both groups of patients (ACC and $\mathrm{CCC}$ ) an increase in the expression of S100A8 protein was linearly paralleled by a significant increase in the expression of S100A9 protein (Figures 6 and 7). 


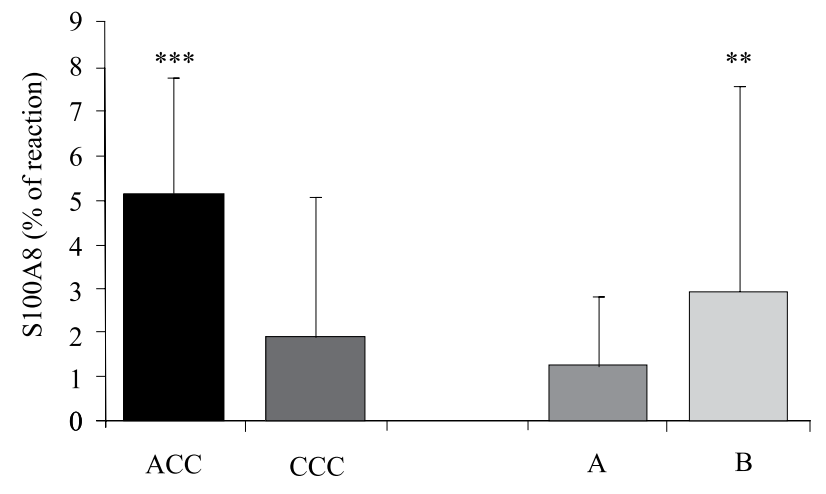

Figure 2. Comparison between semiquantitative immunohistochemical S100A8 protein expression in gallbladder mucosa in patients with acute (ACC) and chronic (CCC) cholecystitis and between younger (A) and older (B) patients with chronic cholecystitis; ${ }^{* *} \mathrm{p}<0.01 ;{ }^{* * *} \mathrm{p}<0.001$

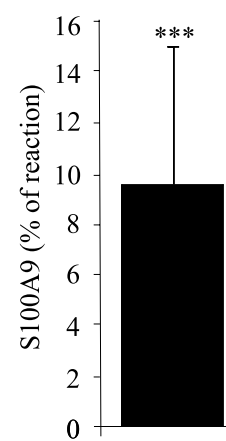

$\mathrm{ACC}$

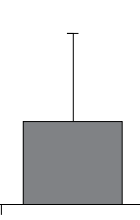

$\mathrm{CCC}$

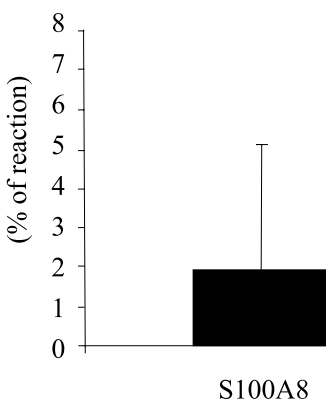

S100A8
$\mathrm{CCC}$

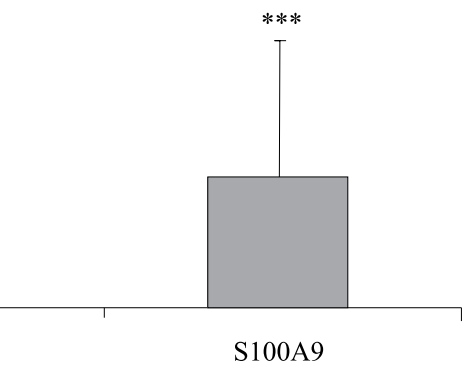

Figure 5. Immunohistochemical expression of family S100 proteins in the gallbladder mucosa in CCC group; ${ }^{* * *} \mathrm{p}<0.001$

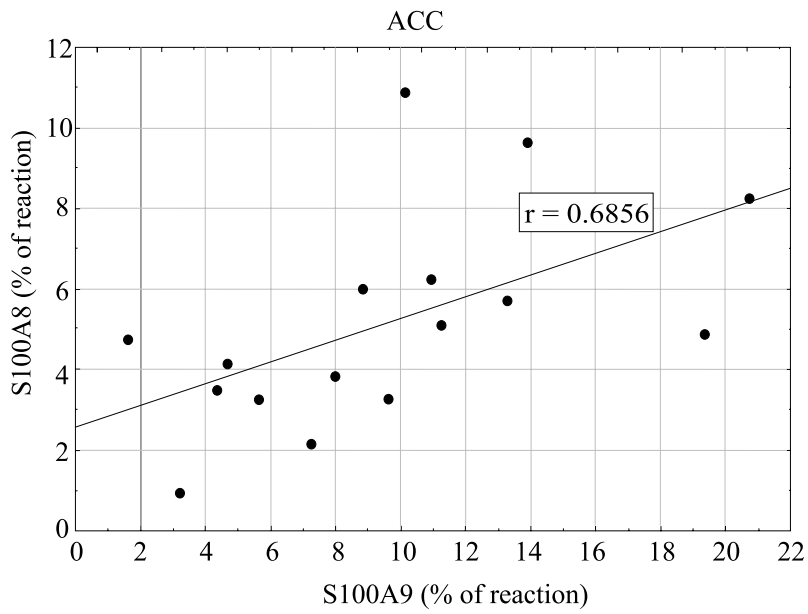

Figure 6. Positive Pearson's correlation between S100A8 and S100A9 proteins immunohistochemical expression in the acute calculous cholecystitis (ACC) group $(\mathrm{p}<0.05)$;

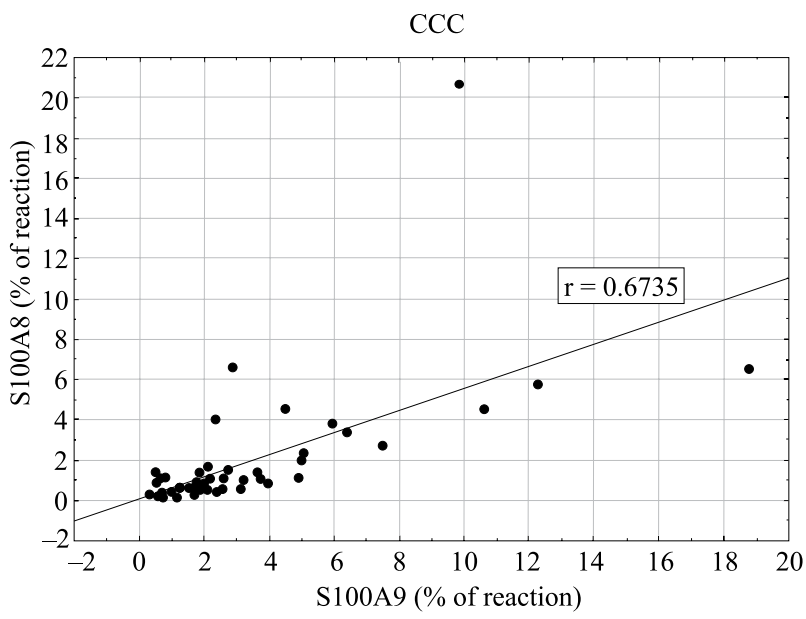

Figure 7. Positive Pearson's correlation between S100A8 and S100A9 proteins immunohistochemical expression in chronic calculous cholecystitis $(\mathrm{CCC})$ group $(\mathrm{p}<0.05)$; $\mathrm{r}$ - correlation coefficient

$\mathrm{r}$ - correlation coefficient
Figure 4. Immunohistochemical expression of family S100 proteins in gallbladder mucosa in ACC group; ${ }^{*}$ p $<0.01$
Figure 3. Comparison between semiquantitative immunohistochemical S100A9 protein expression in gallbladder mucosa in patients with acute (ACC) and chronic (CCC) cholecystitis and between younger (A) and older (B) patients with chronic cholecystitis; $* * * \mathrm{p}<0.001$
S100A8

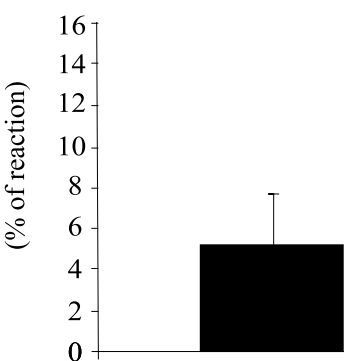

ACC

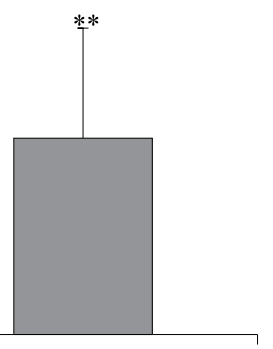

S100A9 
Table 1. Spearman's rank indices for correlation between S100 family proteins immunohistochemical expression and selected clinical data in patients with cholelithiasis

\begin{tabular}{|l|l|l|l|l|l|l|l|l|l|}
\hline & Group & $\begin{array}{l}\text { Age } \\
\text { (years) }\end{array}$ & $\begin{array}{l}\text { Thickness of gallblad- } \\
\text { der's wall }[\mathbf{m m}]\end{array}$ & G1* & G2* & G1+G2* & $\begin{array}{l}\text { BMI } \\
{\left[\mathbf{k g} / \mathbf{m}^{2}\right]}\end{array}$ & $\begin{array}{l}\text { Number of } \\
\text { stones* }\end{array}$ & $\begin{array}{l}\text { WBC } \\
{[\mathbf{x 1 0 9} / \mathbf{L}]}\end{array}$ \\
\hline \multirow{2}{*}{$\begin{array}{l}\text { S100A8 }- \\
\text { protein type }\end{array}$} & ACC & 0.348 & -0.484 & 0.301 & $\mathbf{0 . 5 1 2}$ & 0.450 & 0.159 & 0.495 & -0.147 \\
\cline { 2 - 10 } & CCC & 0.252 & 0.192 & 0.269 & $\mathbf{0 . 3 6 0}$ & $\mathbf{0 . 3 8 4}$ & $\mathbf{0 . 3 2 2}$ & $-\mathbf{0 . 4 1 8}$ & $\mathbf{0 . 3 0 0}$ \\
\hline \multirow{2}{*}{$\begin{array}{l}\text { S100A9 }- \\
\text { protein type }\end{array}$} & ACC & 0.431 & -0.362 & 0.287 & $\mathbf{0 . 5 7 0}$ & 0.453 & -0.282 & 0.485 & -0.171 \\
\cline { 2 - 10 } & CCC & -0.014 & 0.157 & 0.088 & $\mathbf{0 . 3 2 2}$ & 0.239 & 0.127 & $-\mathbf{0 . 3 3 9}$ & 0.235 \\
\hline
\end{tabular}

ACC - acute calculous cholecystitis; CCC — chronic calculous cholecystitis. G1 — grade of lamina propria inflammation (0-3); G2 - grade of muscularis externa/adventitia inflammation (0-3); $\mathrm{G} 1+\mathrm{G} 2$ - total grading in gallbladder wall; BMI - body mass index.

Bold numbers correspond to $\mathrm{r}$ coefficients for which $\mathrm{p}<0.05$; * parameters were appraised semiquantitatively as described in Material and methods

\section{Relationships between S100A8/S100A9 tissue expression and selected clinical data}

In ACC and CCC groups expression of both S100 proteins positively correlated with inflammatory activity in deeper layers of gallbladder wall (parameter G2) and the expression of S100A8 also manifested such a correlation with grading in the entire wall of gallbladder (parameters $\mathrm{G} 1+\mathrm{G} 2$ ) in patients with CCC. The higher correlation coefficient $(r>0.500)$, characterizing the relationship between expression of the protein and $\mathrm{G} 2$, was noted in the case of S100A8 than in the case of S100A9. Also more significant correlation between expression of both S100 proteins and inflammation activity were detected in the ACC group than in the CCC group (Table 1). Moreover, in the CCC group weak positive correlations were disclosed between tissue expression of S100A8 and BMI of patients and white blood cells (WBC) number. We found also a weak negative correlation between the expression of both $\mathrm{S} 100$ proteins and the number of bile stones (Table 1).

\section{Discussion}

Our study was conducted with the use of two different polyclonal antibodies specific for human S100A8 and S100A9 proteins. The presence of these proteins was observed during both acute and the chronic stage of the gallbladder inflammation in all investigated patients. The IHC reaction presented a distinctly visible product present in the cytoplasm of inflammatory cells (mainly neutrophils, monocytes and macrophages) with the typical phenotype described also by other authors in various organs [5, 6, 24-26]. In the studied gallbladder regions, the inflammatory infiltrates of the immunopositive cells were present in lamina propria, as well as frequently between cells of gallbladder epithelium. Both in the case of S100A8, and S100A9, immunopositive cells have manifested themselves in high numbers also in the lumen of blood vessels. Quantitative analysis of the expression of the two S100 proteins in gallbladder mucosa was performed using own, novel method of spatial visualization [22]. Such approach allowed to detect a significantly higher expression of both S100A8, and S100A9 protein in the gallbladder mucosa of the ACC patients as compared with chronic cholecystitis group, a significant prevalence of S100A9 protein expression over that of S100A8 protein and a more pronounced expression of the S100A9 protein in older patients as compared with the younger ones.

The obtained results of the expression of two S100 proteins seem to be original ones since no data on the subject were found, related to human gallbladder. However, the S100A8/A9 complex represents an important mediator of inflammatory reactions in several other diseases of the alimentary tract [4, $5,8]$. Overexpression of the protein complex was documented in particular in chronic inflammatory diseases of intestines and in human glandular tumours $[2,5]$. Measurements of the serum concentration of these proteins may be helpful in monitoring activity of the disease or a response to treatment [4]. In the diseases of the alimentary tract the S100A8/A9 complex („faecal” calprotectin) has been recognized as a marker of acute inflammatory states and assumed to reflect activation of phagocytes [13]. Calgranulins are thought to be released from the cytoplasm of dead neutrophils and to penetrate liquid contents of an abscess [27] or the wall of the gastrointestinal tract so that they may be detected in stools [13].

Expression of the S100A8 and S100A9 proteins in phagocytes of intestinal inflammatory infiltrates was also observed by other investigators by the use of immunocytochemical techniques [14]. The inflammatory-altered parts of the intestinal wall in various diseases of human alimentary tract also demonstrated a much higher contents of S100A8 and S100A9 (and S100A12) proteins than the control mucosa $[5,15$, 
16]. Measurements of the S100A8/A9 complex levels in stools were proposed to provide a marker useful in differential diagnosis of alimentary tract diseases, also in children [28].

Our studies indicate that both S100A8 and S100A9 proteins are present in the gallbladder mucosa in higher amounts in acute cholecystitis than in chronic disease. Their principal cellular source are neutrophils but the proteins may also be produced by activated monocytes and macrophages and several other cell types, as it was demonstrated in the inflammation of various organs [5, 6, 24-26]. Probably, they take an active part in starting an acute inflammation under effect of cellular stress [5, 8]. In acute inflammation (also in our patients) the increased number of mononuclear cells within inflammatory infiltrates was found, which made basis for the higher synthesis of calgranulins by the higher number of cells with phagocytic properties. Apart from neutrophils, the inflammatory infiltrates contained also numerous activated monocytes and macrophages. It was suggested that the S100 family proteins could be produced and secreted by the activated cells of the inflammatory infiltrates [5]. The high correlation indices between reciprocal expression of the two proteins both in acute and in chronic cholecystitis reported in our study support this notion. We found particularly high positive correlations between expression of the two S100 proteins and inflammatory activity in the gallbladder wall (parameter G2) in acute cholecystitis patients. The result indicates that particularly in acute calculous cholecystitis tissue expression of calgranulins may serve as a marker of inflammatory activity.

Some authors proposed that S100A8/S100A9 complex plays an important role at the chronic phase of inflammation since its overexpression was demonstrated in many chronic human diseases $[2,4,5,8,26]$. The suggested mechanisms of the extracellular activities of S100A8/A9 complex involve stimulation of neutrophil and monocyte migration and increased expression of integrins. The involvement of the S100A9 was shown to increase macrophages' migration and activation of their functions, induction of inflammation-associated pain and induction of arteriosclerotic lesions in blood vessels [6]. A direct antibacterial and antimycotic activity of calgranulins was demonstrated in cell culture [7]. Such activity is supposed to reflect inhibition of bacterial metalloproteinases, promotion of neutrophil migration to sites of inflammation and augmentation of granulocytes' phagocytic ability [28].

It should be recalled that the role of $\mathrm{S} 100$ proteins in the pathogenesis of cholelithiasis has not been discussed till now, thus our study provides first original data on this subject. However, it was reported that a positive tissue reaction for $\mathrm{S} 100 \mathrm{~A} 4$ protein was associated with a worsened prognosis of the gallbladder cancer [19].

Studies on the role of S100 family proteins should be continued with demonstration of colocalization of the proteins with other markers of inflammatory infiltrate cells at various stages of inflammation and/or estimation of serum concentration of the proteins in calculous cholecystitis. Further investigations should also deal with the role of the expression of the S100 protein group in gallbladder carcinogenesis.

Summing up, our results indicated close relationship between S100A8 and S100A9 proteins in their proinflammatory functions. Positive correlation between the expression of S100A8 protein in the chronic cholecystitis group and in the group of older patients and total grading (G1+G2) and number of white blood cells count (WBC), would suggest role of S100A8 particularly at the chronic stage of cholecystitis. The results suggest that the increased local expression of each one of the studied S100 proteins may provide an useful index of local inflammatory activity in calculous cholecystitis.

\section{Acknowledgements}

The first author received grant within Subaction 8.2.2. "Regional Strategies of Innovation", Action 8.2 "Transfer of knowledge", Priority VIII "Regional Staff of Management", Operative Programme of Human Capital, co-financed by the European Social Fund of the European Union and by the state budget.

\section{References}

1. Dale I, Fagerhol MK, Naesgaard I. Purification and partial characterization of a highly immunogenic human leukocyte protein, the L1 antigen. Eur J Biochem. 1983;134:1-6.

2. Gebhardt C, Németh J, Angel P, Hess J. S100A8 and S100A9 in inflammation and cancer. Biochem Pharmacol. 2006;72:1622-1631.

3. Srikrishna G. S100A8 and S100A9. New insights into their roles in malignancy. J Innate Immun. 2012;4:31-40.

4. Foell D, Frosch M, Sorg C, Roth J. Phagocyte-specific calcium-binding S100 proteins as clinical laboratory markers of inflammation. Clin Chim Acta. 2004;344:37-51.

5. Foell D, Wittkowski H, Ren Z, et al. Phagocyte-specific S100 proteins are released from affected mucosa and promote immune responses during inflammatory bowel disease. J Pathol. 2008;216:183-192.

6. Goyette J, Geczy CL. Inflammation-associated S100 proteins: new mechanisms that regulate function. Amino Acids. 2011;41:821-842.

7. Hsu K, Champaiboon C, Guenther BD et al. Anti-infective protective properties of S100 calgranulins. Antiinflamm Antiallergy Agents Med Chem. 2009;8:290-305.

8. Foell D, Wittkowski H, Vogl T, Roth J. S100 proteins expressed in phagocytes: a novel group of damage-associated molecular pattern molecules. J Leukoc Biol 2007;81:28-37.

9. Edgeworth J, Gorman M,Bennett R, Freemont P,Hogg N. Identification of $\mathrm{p} 8,14$ as a highly abundant heterodimeric calcium binding protein complex of myeloid cells. JBiol Chem. 1991;266:7706-7713. 
10. Robinson MJ, Tessier P, Poulsom R, Hogg N. The S100 affinity to heparin and heparan sulfate glycosaminoglycans on endothelial cells. J Biol Chem. 2002;277:3658-3665.

11. Vogl T, Tenbrock K, Ludwig S et al. Mrp8 and Mrp14 are endogenous activators of Toll-like receptor 4, promoting lethal, endotoxin-induced shock. Nat Med. 2007;13:1042-1049.

12. Striz I, Trebichavskỳ I. Calprotectin - a pleiotropic molecule in acute and chronic inflammation. Physiol Res. 2004;53:245-253.

13. Manolakis AC, Kapsoritakis AN, Tiaka EK, Potamianos SP. Caprotectin, calgranulin C, and other members of the $s 100$ protein family in inflammatory bowel disease. Dig Dis Sci. 2011;56:1601-1611.

14. Lugering N, Stoll R, Kucharzik T et al. Immunohistochemical distribution and serum levels of the $\mathrm{Ca}(2+)$-binding proteinsMRP8, MRP14 and their heterodimeric form MRP8/14 in Crohn's disease. Digestion. 1995;65:406-414.

15. Leach ST, Yang Z, Messina I et al. Serum and mucosal S100 proteins, calprotectin (S100A8/S100A9) and S100A12, are elevated at diagnosis in children with inflammatory bowel disease. Scand J Gastroenterol. 2007;42:1321-1331.

16. Leach ST, Mitchell HM, Geczy CL, Sherman PM, Day AS. S100 calgranulin proteins S100A8, S100A9 and S100A12 are expressed in the inflamed gastric mucosa of Helicobacter pylori-infected children. Can J Gastroenterol. 2008;22:461-464.

17. Esposito G, Cirillo C, Sarnelli G et al. Enteric glial-derived S100B protein stimulates nitric oxide production in celiac disease. Gastroenterology. 2007;133:918-925.

18. Stulik J, Osterreicher J, Koupilová K et al. The analysis of S100A9 and S100A8 expression in matched sets of macroscopically normal colon mucosa and colorectal carcinoma: the S100A9 and S100A8 positive cells underlie and invade tumor mass. Electrophoresis. 1999;20:1047-1054.

19. Nakamura T, Ajiki T, Murao S et al. Prognostic significance of S100A4 expression in gallbladder cancer. Int J Oncol. 2002;20:937-941.

20. Hsu SM, Raine L, Fanger H. Use of avidin-biotin-peroxidase complex $(\mathrm{ABC})$ in immunoperoxidase techniques: a compari- son between $\mathrm{ABC}$ and unlabeled antibody (PAP) procedures. J Histochem Cytochem. 1981;29:577-580.

21. Kasprzak A, Zabel M, Biczysko W et al. Expression of cytokines (TNF- $\alpha$, IL- $1 \alpha$, and IL-2) in chronic hepatitis C: comparative hybridocytochemical and immunocytochemical study in children and adult patients. J Histochem Cytochem. 2004;52:29-38.

22. Kaczmarek E, Strzelczyk R. From two to three-dimensional visualisation of structures in light and confocal microscopy - applications for biomedical studies. In: Mendez-Vilas A, Labajos-Broncano L (eds.). Current issues on multidisciplinary microscopy research and education. FORMATEX microscopy book series no. II Formatex Research Centre: Badajoz; 2005:289-295.

23. Kasprzak A, Malkowski W, Helak-Lapaj C et al. Polysaccharides and mucin 5AC (MUC5AC) expression in gallbladder mucosa of young patients with gallstones as evaluated by spatial visualization and quantification. Folia Histochem Cytobiol. 2010;48:646-657.

24. Ryckman C, Vandal K, Rouleau P, Talbot M, Tessier PA. Proinflammatory activities of S100: proteins S100A8, S100A9, and S100A8/A9 induce neutrophil chemotaxis and adhesion. J Immunol. 2003;170:3233-3242.

25. Roth J, Vogl T, Sorg C, Sunderkotter C. Phagocyte-specific S100 proteins: a novel group of proinflammatory molecules. Trends Immunol. 2003;24:155-158.

26. Foell D, Wittkowski H, Roth J. Monitoring disease activity by stool analysis: from occult blood to molecular markers of intestinal inflammation and damage. Gut. 2009;58:859-868.

27. Kocher M, Kenny PA, Farram E, Abdul Majid KB, Finlay -Jones JJ, Geczy CL. Functional chemotactic factor CP-10 and MRP-14 are abundant in murine abscesses. Infect Immun. 1996;64:1342-1350.

28. Szarszewski A, Korzon M, Kurlenda J et al. Faecal calprotectin: a new acute inflammatory reactant in the diagnosis of childhood Inflammatory Bowel Disease - our experience. Med Sci Monit. 2003;9(Suppl. 4):11-13.

Submitted: 6 May, 2013

Accepted after reviews: 21 June, 2013 